\title{
Clinical Study of Laparoscopy Combined with Choledochoscope in the Treatment of Cholecystolithiasis Combined with Choledocholithiasis
}

\author{
B. HUANG, YIFAN CHEN, MIN ZHAI, ANCHENG QIN, JIANWU WU, Y. LU, XINWEI JIANG AND Z. QIAO*
}

Department of Hepatobiliary Surgery, Suzhou Municipal Hospital, The Affiliated Suzhou Hospital of Nanjing Medical University, No. 26, Daoqian Street, Suzhou, Jiangsu 215000, China

\section{Huang et al.: Clinical Effect of Combined Laparoscopic and Choledochoscopic Surgery}

To observe the clinical effect of combined laparoscopic and choledochoscopic surgery for gallbladder combined with common bile duct stones. A total of 102 patients with gallbladder stones combined with common bile duct stones admitted to our hospital between January 2017 and December 2020 were selected and randomly divided into 51 cases in the study group and 51 cases in the control group. The control group was treated with laparoscopic gallbladder stones treatment and the trial group was treated with laparoscopic combined with choledochoscopic surgery. The surgery-related indexes, serum inflammatory indexes and postoperative complications of patients in the two groups were compared, as well as the treatment efficiency. After treatment, the intraoperative bleeding, average postoperative hospitalization time, $24 \mathrm{~h}$ postoperative visual analogue scale score and time to first postoperative venting of patients in the laparoscopic group were significantly better than those in the control group, but the operative time was significantly more than that in the control group and the difference was statistically significant $(p<0.05)$. Before surgery, there was no significant difference between the serum procalcitonin and highsensitivity $C$-reactive protein levels of the two groups ( $\mathbf{p}>0.05) ; 1 \mathrm{~d}$ after surgery, the serum procalcitonin and high-sensitivity $C$-reactive protein levels of the two groups were lower than those before surgery and the study group was significantly lower than the control group and the difference was statistically significant $(\mathbf{p}<\mathbf{0 . 0 5})$. There was no statistically significant difference between the two groups in terms of total treatment effectiveness $(\mathrm{p}>\mathbf{0 . 0 5})$. The total complication rate of the test group was lower than that of the control group $(\mathbf{p}<\mathbf{0 . 0 5})$. The treatment of patients with gallbladder combined with common bile duct stones by laparoscopy combined with choledochoscopy has better clinical treatment effect than laparoscopic gallbladder stones treatment and has the advantages of less trauma, faster postoperative recovery and less inflammatory reaction, which is worthy of clinical promotion and application.

Key words: Laparoscopy, cholangioscopy, gallbladder stones, bile duct, clinical efficacy

Cholecystolithiasis and choledocholithiasis are common biliary tract diseases, with high incidence rate. The main symptoms are pain in the upper right abdomen, jaundice and abnormal liver function. Severe cases may cause septic shock, which poses a serious threat to life safety ${ }^{[1]}$. Surgery is an important way to treat cholecystolithiasis combined with choledocholithiasis, but traditional laparoscopic gallbladder stones treatment has great trauma, easy to damage the function of duodenal papillary sphincter, resulting in poor postoperative recovery of patients ${ }^{[2]}$. In recent years, with the rapid development of endoscopic technology, laparoscopy and choledochoscopy have been widely used in the clinical treatment process $^{[3]}$, the combined application of the two has become the mainstream scheme for the treatment of cholecystolithiasis combined with choledocholithiasis $^{[4]}$. However, compared with simple cholecystolithiasis, the condition of cholecystolithiasis combined with choledocholithiasis is more complex. Although the effect of combined application has been clear, its safety and applicability are still unclear sex remains to be studied. This study analyzes the effect and safety of laparoscopy combined with

*Address for correspondence

E-mail: zmq800908@163.com 
choledochoscope in the treatment of cholecystolithiasis combined with choledocholithiasis. A total of 102 patients with cholecystolithiasis and choledocholithiasis admitted to our hospital from January 2017 to December 2020 were selected. Inclusive criteria: All patients were diagnosed by computed tomography (CT), magnetic resonance imaging (MRI), cholangiopancreatography and B-ultrasound; Patients had typical symptoms such as abdominal pain, chills, high fever and jaundice; all patients signed informed consent. Exclusion criteria: Patients with coagulation dysfunction; Patients with heart, liver, kidney dysfunction; Patients with acute pancreatitis, acute cholangitis; Patients with intrahepatic and extrahepatic bile duct stenosis. This study was approved by the medical ethics committee of our college. All patients were randomly divided into control group $(n=51)$ and study group $(n=51)$. There were 30 males and 21 females in the control group, with an average age of (49.40 \pm 5.25$)$ y (range, 31-67 y). There were 31 males and 20 females in the observation group, with an average age of (50.86 \pm 5.30$)$ y (range, 32-69 y). There was no significant difference in general information between the two groups $(\mathrm{p}>0.05)$. The control group received laparoscopic gallbladder stones treatment: after general anesthesia, trocar puncture was performed on the right subcostal abdominal wall and a non-invasive gallbladder gripper was inserted. The base of the gallbladder was lifted with a straight needle suture of No. 1 silk thread to fully expose the gallbladder. An aspirator was placed at the lower edge of the incision. According to the size of the calculi, the gallbladder base was cut open with an electric hook and then the calculi were removed with a bile duct. After the calculi were removed, the gallbladder was introduced into a sputum suction tube and the gallbladder was repeatedly flushed. During the operation, B-ultrasound was used to check whether all the stones were removed. Finally, the gallbladder incision was sewn with continuous hemlock with the whole layer of absorbable thread, and the gallbladder was brought back into the abdomen and the traction thread that raised the gallbladder was cutoff. After the examination of biliary fistula and active bleeding, the abdominal cavity was closed and the operation ended. The study group received laparoscopy combined with choledochoscopy surgery: the anesthesia method was the same as that of the control group in pneumoperitoneum, laparoscopy and operating instruments were placed in the operating hole. The triangle of gallbladder was identified by laparoscopy. The cystic duct was dissected from the neck of gallbladder. The common bile duct and common hepatic duct were fully exposed. The distal cystic duct was clamped and the anterior wall of cystic duct was cut. The right angle forceps were inserted into the cystic duct to expand the incision. The urinary catheter and choledochoscope were inserted and the stones with diameter less than $10 \mathrm{~mm}$ were removed with stone basket; the stones with diameter more than $10 \mathrm{~mm}$ were removed after lithotripsy. Normal saline was used to flush the biliary tract. After choledochoscopy, the anterior wall of gallbladder was sutured, the cystic duct was cut off, the gallbladder was removed and the drainage tube was placed. The operation related indexes of the two groups were compared, including operation time, postoperative hospital stay, postoperative first exhaust time and postoperative $24 \mathrm{~h}$ pain score. The degree of pain was scored by visual analogue scale (VAS). The higher the score, the stronger the pain; the serum inflammatory indexes including interleukin-6 (procalcitonin) and high-sensitivity C-reactive protein (hs-CRP) were compared between the two groups before and after operation. Procalcitonin was detected by radioimmunoassay and hs-CRP by immunoturbidimetry; Complications: including reflux esophagitis, incision infection, bile leakage; Clinical efficacy criteria: imaging examination results showed no residual stones, clinical symptoms disappeared, jaundice subsided as markedly effective; imaging examination results showed a small amount of residual stones, clinical symptoms improved as effective; did not meet the above criteria for invalid ${ }^{[4]}$. The total effective rate of treatment=significant efficiency+effective rate. All data of this study were analyzed by statistical package for the social sciences (SPSS) 20.0 software. The count data was expressed by percentage, using chi square test; the measurement data was expressed by $\mathrm{x} \pm \mathrm{s}$, using $\mathrm{t}$ test; $\mathrm{p}<0.05$ was considered to be statistically significant. The intraoperative blood loss, postoperative average hospital stay, postoperative $24 \mathrm{~h}$ VAS score and postoperative first exhaust time of the study group were significantly better than those of the control group, but the operation time was significantly longer than that of the control group $(p<0.05)$, as shown in Table 1. Before operation, there was no significant difference in serum procalcitonin and hs-CRP levels between the two groups $(p>0.05) ; 1 \mathrm{~d}$ after operation, the serum procalcitonin and hs-CRP levels of the two groups were lower than before operation and the study group was significantly lower than the control group $(p<0.05)$, as 
shown in Table 2. The total incidence of complications in the study group was lower than that in the control group, and the difference was statistically significant $(\mathrm{p}<0.05)$, as shown in Table 3 . There was no significant difference in the total effective rate between the two groups ( $p>0.05)$, as shown in Table 4 . In recent years, due to the obvious changes of diet structure in our country, the incidence rate of gallstone and choledocholithiasis has also increased. If not treated effectively, it will pose a serious threat to the health of the patients ${ }^{[5]}$. The main method of clinical treatment of gallstone and choledocholithiasis is to take stones by operation. The open operation requires " $t$ " tube drainage, which is not conducive to the early postoperative bed walking, increased risk of intestinal adhesion and patients are afraid to cough hard and the risk of pulmonary infection increase ${ }^{[6]}$. Elderly patients often complicated with a variety of underlying diseases, surgical tolerance decreased, surgical risk increased. Choledochoscopy and laparoscopy technology is gradually mature, clinical treatment of this disease more choose multi mirror combination, laparoscopy combined with choledochoscopy exploration and lithotomy has been widely used ${ }^{[7-9]}$. This study results showed that the amount of blood loss, average postoperative hospital stay, postoperative $24 \mathrm{~h}$ VAS score, postoperative first exhaust time of the study group were significantly better than those of the control group, while the operation time was significantly longer than that of the control group, indicating that the short term effect of laparoscopy combined with choledochoscope in the treatment of gallbladder complicated with choledocholithiasis is better than that of laparotomy. But the operation time is longer, which may be related to the complexity of laparoscopy combined with choledochoscope and the learning curve of surgeons ${ }^{[10]}$. There was no significant difference in serum procalcitonin and hs-CRP levels between the two groups before operation; on the 1 st d after operation, the serum procalcitonin and hs-CRP levels of the two groups were lower than before operation and the study group was significantly lower than the control group, suggesting that the inflammatory response caused by laparoscopy combined with choledochoscope was smaller, which was related to the less stimulation of abdominal cavity and the lower stress response caused by minimally invasive surgery. These data suggest that laparoscopic combined with choledochoscopy does not increase the risk of infection in patients with cholecystolithiasis and choledocholithiasis ${ }^{[11,12]}$. Our results showed that there was no significant difference in the total effective rate between the two groups and the total incidence of complications in the study group was lower than that in the control group, suggesting that laparoscopy combined with choledochoscopy in the treatment of cholecystolithiasis combined with choledocholithiasis can achieve the same stone removal effect as laparoscopic gallbladder stones treatment, with high safety and in line with clinical needs. Laparoscopy combined with choledochoscopy can make full use of the physiological channels of patients and the operation is not destructive to the biliary tract, which can retain the normal physiological function of biliary $\operatorname{tract}^{[13]}$. In addition, the operation is accurate, less blood loss, less impact on the abdominal cavity, postoperative intestinal peristalsis function can be recovered in a short time, patients can get out of bed as soon as possible ${ }^{[14]}$. However, for patients with gallbladder obstruction, stones are too large; it is not suitable to use laparoscopy for treatment. Secondly, long-term biliary operation is easy to cause nipple edema and spasm, and the probability of bile leakage is high $^{[15,16]}$. In conclusion, laparoscopy combined with choledochoscopy in the treatment of cholecystolithiasis combined with choledocholithiasis has significant effect, high safety, and can effectively reduce postoperative pain and inflammatory reaction.

TABLE 1: COMPARISON OF OPERATION INDEXES BETWEEN THE TWO GROUPS

\begin{tabular}{lccccc}
\hline Group & $\begin{array}{c}\text { Intraoperative } \\
\text { blood loss }(\mathrm{ml})\end{array}$ & $\begin{array}{c}\text { Operation time } \\
(\mathrm{min})\end{array}$ & $\begin{array}{c}\text { Postoperative } \\
\text { hospital stay }(\mathrm{d})\end{array}$ & $\begin{array}{c}\text { The first exhaust } \\
\text { time after } \\
\text { operation }(\mathrm{h})\end{array}$ & $\begin{array}{c}\text { VAS score 24 h } \\
\text { after operation }\end{array}$ \\
\hline Control group & $102.6 \pm 11.2$ & $85.3 \pm 6.7$ & $7.7 \pm 2.7$ & $32.4 \pm 3.7$ & $3.1 \pm 0.8$ \\
Study group & $54.8 \pm 9.6^{*}$ & $113.1 \pm 12.4^{*}$ & $7.4 \pm 1.1^{*}$ & $20.9 \pm 2.3^{*}$ & $1.7 \pm 0.5^{*}$ \\
\hline
\end{tabular}

Note: compared with the control group, ${ }^{*} \mathrm{p}<0.05$ 
www.ijpsonline.com

TABLE 2: COMPARISON OF SERUM INFLAMMATORY FACTORS BETWEEN TWO GROUPS BEFORE AND AFTER OPERATION

\begin{tabular}{|c|c|c|c|c|c|}
\hline \multirow{2}{*}{ Group } & \multirow{2}{*}{$\mathrm{n}$} & \multicolumn{2}{|c|}{ Procalcitonin (ng/l) } & \multicolumn{2}{|c|}{ hs-CRP (mg/l) } \\
\hline & & Before operation & $1 \mathrm{~d}$ after operation & Before operation & $1 \mathrm{~d}$ after operation \\
\hline Control group & 51 & $60.5 \pm 28.4$ & $47.2 \pm 13.6^{\#}$ & $28.2 \pm 3.1$ & $21.9 \pm 3.3^{\#}$ \\
\hline Study group & 51 & $61.7 \pm 29.8$ & $30.6 \pm 10.3^{* \#}$ & $28.5 \pm 3.2$ & $14.8 \pm 3.5^{* \#}$ \\
\hline
\end{tabular}

Note: compared with before treatment, ${ }^{\#} p<0.05$; compared with the control group, ${ }^{*} p<0.05$

TABLE 3: COMPARISON OF COMPLICATIONS BETWEEN THE TWO GROUPS

\begin{tabular}{|c|c|c|c|c|}
\hline Group & Reflux esophagitis & Incision infection & Bile leakage & Total incidence \\
\hline Study group & $1(1.96)$ & $0(0.00)$ & $2(3.92)$ & 5.88 \\
\hline Control group & $4(7.68)$ & $2(3.92)$ & $4(7.68)$ & 19.6 \\
\hline$\chi^{2} / p$ & \multicolumn{4}{|c|}{$10.83 /<0.001$} \\
\hline
\end{tabular}

TABLE 4: COMPARISON OF CLINICAL EFFICACY BETWEEN THE TWO GROUPS

\begin{tabular}{lcccc}
\hline Group & Markedly effective & Effective & Ineffective & Total effective rate \\
\hline Study group & $48(94.12)$ & $2(3.92)$ & $1(1.96)$ & 98.04 \\
Control group & $45(88.40)$ & $4(7.68)$ & $2(3.92)$ & 96.08 \\
$\chi^{2} / \mathrm{p}$ & & $0.76 />0.05$ & & \\
\hline
\end{tabular}

\section{Author's contributions:}

This work was supported by the Suzhou Municipal Hospital, The Affiliated Suzhou Hospital of Nanjing Medical University.

\section{Conflict of interests:}

The authors report no conflicts of interest.

\section{REFERENCES}

1. Williams EJ, Green J, Beckingham I, Parks R, Martin D, Lombard M. Guidelines on the management of common bile duct stones (CBDS). Gut 2008;57(7):1004-21.

2. Frossard JL, Morel PM. Detection and management of bile duct stones. Gastrointest Endosc 2010;72(4):808-16.

3. Baillie J, Testoni PA. Same-day laparoscopic cholecystectomy and ERCP for choledocholithiasis. Gastrointest Endosc 2016;84(4):646-8.

4. Tinoco R, Tinoco A, El-Kadre L, Peres L, Sueth D. Laparoscopic common bile duct exploration. Ann Surg 2008;247(4):674-9.

5. Wandling MW, Hungness ES, Pavey ES, Stulberg JJ, Schwab $\mathrm{B}$, Yang $\mathrm{AD}$, et al. Nationwide assessment of trends in choledocholithiasis management in the United States from 1998 to 2013. JAMA Surg 2016;151(12):1125-30.

6. Mattila A, Luhtala J, Mrena J, Kautiainen H, Kellokumpu I. An audit of short-and long-term outcomes after laparoscopic removal of common bile duct stones in Finland. Surg Endosc 2014;28(12):3451-7.

7. Zhang WJ, Xu GF, Wu GZ, Li JM, Dong ZT, Mo XD. Laparoscopic exploration of common bile duct with primary closure versus T-tube drainage: a randomized clinical trial. J Surg Res 2009;157(1):e1-5.
8. Wu X, Yang Y, Dong P, Gu J, Lu J, Li M, et al. Primary closure versus T-tube drainage in laparoscopic common bile duct exploration: a meta-analysis of randomized clinical trials. Langenbecks Arch Surg 2012;397(6):909-16.

9. Zhu QD, Tao CL, Zhou MT, Zheng PY, Shi HQ, Zhang QY. Primary closure versus T-tube drainage after common bile duct exploration for choledocholithiasis. Langenbecks Arch Surg 2011;396(1):53-62.

10. Khaled YS, Malde DJ, de Souza C, Kalia A, Ammori BJ. Laparoscopic bile duct exploration via choledochotomy followed by primary duct closure is feasible and safe for the treatment of choledocholithiasis. Surg Endosc 2013;27(11):4164-70.

11. Hua J, Lin S, Qian D, He Z, Zhang T, Song Z. Primary closure and rate of bile leak following laparoscopic common bile duct exploration via choledochotomy. Dig Surg 2015;32(1):1-8.

12. Koch M, Garden OJ, Padbury R, Rahbari NN, Adam R, Capussotti L, et al. Bile leakage after hepatobiliary and pancreatic surgery: a definition and grading of severity by the International Study Group of Liver Surgery. Surgery 2011;149(5):680-8.

13. Chan DS, Jain PA, Khalifa A, Hughes R, Baker AL. Laparoscopic common bile duct exploration. Br J Surg 2014;101(11):1448-52.

14. Lee JS, Yoon YC. Laparoscopic common bile duct exploration using V-Loc suture with insertion of endobiliary stent. Surg Endosc 2016;30(6):2530-4.

15. Köhler G, Mayer F, Lechner M, Bittner R. Small bowel obstruction after TAPP repair caused by a self-anchoring barbed suture device for peritoneal closure: case report and review of the literature. Hernia 2015;19(3):389-94. 
16. Topal B, Vromman K, Aerts R, Verslype C, Van Steenbergen W, Penninckx F. Hospital cost categories of one-stage versus two-stage management of common bile duct stones. Surg Endosc 2010;24(2):413-6.
This is an open access article distributed under the terms of the Creative Commons Attribution-NonCommercial-ShareAlike 3.0 License, which allows others to remix, tweak, and build upon the work non-commercially, as long as the author is credited and the new creations are licensed under the identical terms

This article was originally published in a special issue, "Diagnostic and Therapeutic Advances in Biomedical Research and Pharmaceutical Sciences"

Indian J Pharm Sci 2021:83(5) spl issue "6-10" 